\title{
Astrobiology Markers Predicted by Synthetic Metabolisms Generated by Stochastic Evolutionary Network Expansion and Reduction
}

\author{
ANDISHEH DADASHI ${ }^{1,2}$, ERIC LIBBY ${ }^{5,6}$, G. MATTHEW
}

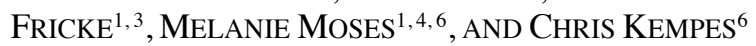

${ }^{1}$ Department of Computer Science, University of New Mexico, Albuquerque, NM 87131, USA

${ }^{2}$ Division of Mathematics, Engr. and CS., UNM-Valencia, Los Lunas, NM 87031, USA (andisheh@unm.edu)

${ }^{3}$ Center for Advanced Research Computing, Albuquerque, NM 87131, USA (mfricke@unm.edu)

${ }^{4}$ Biology Dept., UNM, Albuquerque, NM 87131, USA (melaniem@unm.edu)

${ }^{5}$ Dept. of Mathematics and Mathematical Statistics, Umeå Univ., 90187 Umeå, Sweden (eric.libby@umu.se)

${ }^{6}$ Santa Fe Inst., Santa Fe, NM 87501, USA

(ckempes@santafe.edu)

Detection of astrobiological molecular markers is informed by modeling synthetic metabolisms in potential non-terrestrial molecular environments. We use genetic algorithms to evolve synthetic metabolisms that can survive in these test environments. We draw our universe of available reactions from the KEGG database (Escherichia coli K-12) and use flux balance analysis (FBA) as our measure of viability.

Our computational model uses evolutionary algorithms coupled with stochastic expansion and reduction methods to explore metabolic space and generating novel metabolisms.

We investigate the viability of the resulting metabolic networks in 49 aerobic and anaerobic carbon source environments, and report the molecular inputs required and the molecules our synthetic metabolisms put into the environment. These outputs provide information on potential biomarkers given these unique environments.

We also explore the effect of using network expansion vs reduction on the kinds of metabolisms discovered. We report on the similarities and differences that result from using these two methods. We use our model to show the variety of metabolisms that are viable in the environments. We compare the sensitivity of the metabolisms to carbon availability.

Our model allows us to test hypotheses about the environmental conditions required for viability by adding and removing molecules in the environments, in this way we can explore the boundary conditions that define viability.

Using evolutionary methods and flux balance analysis allows the rapid exploration of novel metabolic spaces when compared to current systematic approaches. 